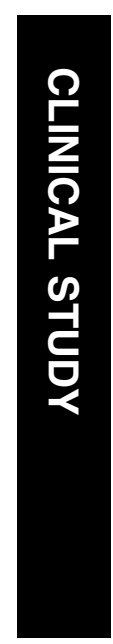

\title{
Effect of illumination on visual function after monofocal and multifocal intraocular lens implantation
}

${ }^{1}$ Ophthalmology Department, Essex County Hospital, Colchester, UK

${ }^{2}$ University Ophthalmology Hospital, Tanta University Hospitals, Tanta, Egypt

Correspondence: MA Elgohary, Essex County Hospital, Colchester CO3 3NB, UK Tel: + 4401206 852166; Fax: +44 1206744742 .

E-mail: m.elgohary@ doctors.org.uk

Received: 30 July 2004; Accepted in revised form: 1 November 2004: Published online: 22 April 2005

Meeting presentation: Part of the data was presented as a poster at the Association for Research in Vision and Ophthalmology (ARVO) annual meeting, Fort Lauderdale, 2003 and at Oxford Annual Congress, Oxford, UK, 2003

Proprietary interests: None
Abstract

Purpose To compare best-corrected visual acuity (BCVA) and contrast sensitivity (CS) under different levels of illumination in patients who had monofocal and multifocal intraocular lenses (IOLs) and to establish the effect of different lighting conditions on vision in the two groups of patients.

Methods We retrospectively reviewed 27 patients who underwent phacoemulsification for age-related cataract and IOL implantation of either monofocal (SI30NB; $\boldsymbol{n}=\mathbf{1 0}, \mathbf{3 7 \%}$ ) or multifocal (SA40N; $n=17,63 \%$ ) IOLs.

Binocular distance and near BCVA and CS were tested using $\log$ MAR and Pelli-Robson charts that were externally illuminated with 20, 200, 400 and 1600 lux, and were compared using repeated-measures analysis of variance. A questionnaire was administered to establish the lighting preference in the two groups and the effect of lighting conditions on their vision.

Results Binocular distance and near BCVA and CS significantly increased with increasing illumination from 20 to 200 lux in the monofocal (mean $=0.04$ vs $-0.07, P=0.006$; 0.37 vs $0.26, P=0.002$ and 1.47 vs $1.60, P=0.01$ ) as well as in the multifocal group (mean $=$ 0.03 vs $-0.12, P<0.001 ; 0.38$ vs $0.23, P<0.001$ and 1.47 vs $1.61, P=0.002)$. No significant difference in BCVA or CS was found between the two groups at any of the four illumination levels. Both groups had similar lighting preference, but $43.8 \%$ of patients in the multifocal group experienced subjective worsening of their vision in bright outdoor lights. Conclusions Distance and near BCVA and CS improve with increasing illumination in
MA Elgohary ${ }^{1,2}$ and $A B$ Beckingsale

patients with monofocal and multifocal IOLs, but remain comparable in the two groups under common levels of indoor illumination. Patients with multifocal IOLs may experience worsening of their vision in bright outdoor lights.

Eye (2006) 20, 144-149. doi:10.1038/sj.eye.6701820; published online 22 April 2005

Keywords: lenses; intraocular; lighting; visual acuity; contrast sensitivity; cataract; phacoemulsification

Introduction

Multifocal intraocular lenses (IOLs) have the advantage of providing useful near and intermediate vision without spectacles and without adversely affecting distance vision. ${ }^{1-5}$ However, they are known to reduce contrast sensitivity (CS) under low-contrast ${ }^{6,7}$ and mesopic conditions, ${ }^{8}$ and to induce photic phenomena such as glare and halo with bright light. ${ }^{5,6,9}$

In daily life, exposure to different levels of illumination is unavoidable and, although visual acuity is known to improve with increasing illumination, ${ }^{10}$ it has not been reported before if, in patients with multifocal IOLs, visual acuity and CS are affected by changing the level of illumination.

The aims of this study were to examine and compare distance and near best-corrected visual acuity (BCVA) and CS in patients who had monofocal and those who had multifocal IOLs under increasing levels of illumination and to establish if the perceived effect of different lighting conditions on vision vary in the two groups. 


\section{Method}

After obtaining approval for the study protocol from the local ethical committee, we retrospectively reviewed 43 consecutive nonrandomised patients who had uneventful bilateral phacoemulsification for age-related cataract and implantation of either monofocal IOLs (AMO, SI30NB; Advanced Medical Optics Ltd) $(n=14)$ or multifocal IOLs (AMO Array, SA40N; Advanced Medical Optics Ltd) $(n=29)$. All the patients had surgery under the care of a single consultant (ABB) and were given the implant type depending on their preference and their life style after discussing with them the potential advantages and disadvantages of each.

Routine clinical examination was carried out to confirm the absence of any fundus pathology that could affect the visual function. Patients were excluded if they had amblyopia (one) or posterior capsular opacification if it reduced the postoperative visual acuity by 1 or more Snellen's lines or if it was more than grade 1 (mild greyish haze of the capsule or lens epithelial cell proliferation if involving the pupillary area) (five). Also, patients with ocular co-morbidity, for example, glaucoma (two), diabetic retinopathy requiring treatment (two), neo-vascular ARMD (two) or retinal pigment epithelial atrophy (four) were excluded.

Prior to examination, all the patients were given a questionnaire that included questions about: spectacle usage, visual symptoms and lighting preferences.

Patients with spectacles wore their distance correction for the distance vision and CS tests and their near add for the near vision test. Binocular distance and near-BCVA were tested using logMAR distance and near charts and CS using Pelli-Robson chart. The tests were performed under dim background illumination to eliminate sources of glare from reflected light. Halogen lamps attached to a rheostat for adjustment of the illumination levels were used to externally illuminate the $\log$ MAR and PelliRobson charts and a light meter (Mavolux 5032C/B; Gossen, Germany) was used to measure the illumination level at the charts and to check it throughout the test. The tests were carried out under four levels of illumination20, 200, 400 and 1600 lux - which were sequentially used to represent four photopic conditions. These levels were chosen guided by the recommended levels of illumination by International Engineering Society (IES) for indoor activities. ${ }^{11}$ The scores of distance and nearvisual acuities and CS were recorded for each patient at each of the illumination levels.

Initial results showed that for a two-sided paired and unpaired $t$-test, a sample size of 10 patients per group would detect a difference of 0.20 of $\log$ MAR visual acuity (10 letters or two lines) with a standard deviation (SD) of
0.15 and six patients per group would detect a difference of 0.3 (6 letters) of CS on the Pelli-Robson chart, with an SD of 0.15 at $80 \%$ power and a significance level of 0.05 . Statistical analysis was carried out using the Statistical Package for Social Science (SPSS) Software (v.9). The median and interquartile range or mean and SD were used for description of the data. Repeated-measures analysis of variance (ANOVA) - with Bonferronicorrected pairwise comparison - was used to compare the visual acuity and CS at the different levels of illumination. Within-subjects factor was the illumination levels and between-subjects factor was the IOL type. A $P$-value of $<0.05$ was considered statistically significant.

\section{Results}

In all, 27 patients were included, 10 of them had monofocal (37\%) and 17 multifocal (63\%) IOLs, of whom 9 and 16, respectively, answered the questionnaire. The patients' clinical features are detailed in Table 1 . There was no statistically significant difference between the two groups in age, sex distribution, IOL power, postoperative spherical equivalent or followup period.

In the monofocal group, distance and near BCVA and CS significantly increased as illumination was increased only from 20 to 200 lux (mean $=0.04$ vs -0.07 ; 0.37 vs 0.26 , and 1.47 vs $1.60 ; P=0.006,0.002$ and 0.01 ). Also, in the multifocal group, distance and near-visual acuity and CS significantly improved by increasing illumination from 20 to 200 lux (mean $=0.03$ vs $-0.12,0.38$ vs 0.23 , and 1.47

Table 1 Clinical features of patients

\begin{tabular}{lcc}
\hline & $\begin{array}{c}\text { Monofocal IOLs } \\
(\mathrm{N}=10)\end{array}$ & $\begin{array}{c}\text { Multifocal IOLs } \\
(\mathrm{N}=17)\end{array}$ \\
\hline Male: Female & $5: 5$ & $9: 8$ \\
& & \\
$\begin{array}{l}\text { Age (years) } \\
\text { Median }\end{array}$ & 75.9 & 77.1 \\
$\quad$ IQR & $74.5-79.7$ & $72.3-79.1$ \\
& & \\
IOL power & \\
$\quad$ Median & 19.5 & 21 \\
$\quad$ IQR & $16.6-22$ & $19-22$ \\
Spherical Equivalent $(D)^{\mathrm{a}}$ & & \\
$\quad$ Median & 0.00 & 0.50 \\
$\quad$ IQR & -2.1 to 0.4 & -0.38 to 0.78 \\
Follow-up (Months) & & \\
$\quad$ Median & 20.8 & 18.5 \\
IQR & $11.1-24.6$ & $10-22.5$ \\
\hline
\end{tabular}

$\mathrm{IQR}=$ interquartile range; $\mathrm{IOL}=$ intraocular lens, $\mathrm{M}=$ male, $\mathrm{F}=$ female. aBoth eyes included. 
Table 2 Comparison of distance and near-logMAR BCVA and Pelli-Robson CS scores at the four levels of illumination in the monofocal (SI30NB) and multifocal (SA40N) IOL groups

\begin{tabular}{|c|c|c|c|c|c|c|c|c|}
\hline & \multicolumn{4}{|c|}{ Monofocal $(\mathrm{n}=10)($ lux $)$} & \multicolumn{4}{|c|}{ Multifocal $(\mathrm{n}=17)($ lux $)$} \\
\hline & 20 & 200 & 400 & 1600 & 20 & 200 & 400 & 1600 \\
\hline \multicolumn{9}{|c|}{ Distance BCVA (logMAR) } \\
\hline Mean (SD) & $0.04(0.13)$ & $-0.07(0.12)$ & $-0.10(0.13)$ & $-0.09(0.13)$ & $0.03(0.22)$ & $-0.12(0.20)$ & $-0.12(0.19)$ & $-0.16(0.16)$ \\
\hline $\mathrm{CI}$ & $-0.05,0.13$ & $-0.16,0.01$ & $-0.19,-0.01$ & $-0.19,0.001$ & $-0.08,0.14$ & $-0.22,-0.02$ & $-0.22,-0.02$ & $-0.24,-0.08$ \\
\hline$P$ & & 0.006 & 0.35 & 0.99 & & $<0.001$ & 0.99 & 0.78 \\
\hline \multicolumn{9}{|c|}{ Near BCVA $(\log M A R)$} \\
\hline Mean (SD) & $0.37(0.15)$ & $0.26(0.12)$ & $0.22(0.08)$ & $0.18(0.11)$ & $0.38(0.15)$ & $0.23(0.12)$ & $0.20(0.13)$ & $0.17(0.11)$ \\
\hline CI & $0.26,0.48$ & $0.18,0.34$ & $0.16,0.28$ & $0.10,0.26$ & $0.30,0.45$ & $0.17,0.29$ & $0.13,0.27$ & $0.11,0.23$ \\
\hline$P$ & & 0.002 & 0.22 & 0.37 & & $<0.001$ & 0.61 & 0.38 \\
\hline \multicolumn{9}{|c|}{ Contrast Sensitivity (Pelli-Robson) } \\
\hline Mean (SD) & $1.47(0.13)$ & $1.60(0.16)$ & $1.60(0.12)$ & $1.66(0.08)$ & $1.47(0.13)$ & $1.61(0.17)$ & $1.63(0.14)$ & $1.65(0.14)$ \\
\hline $\mathrm{CI}$ & $1.38,1.56$ & $1.49,1.71$ & $1.52,1.68$ & $1.60,1.72$ & $1.41,1.54$ & $1.52,1.70$ & $1.56,1.70$ & $1.58,1.72$ \\
\hline$P$ & & 0.01 & 0.99 & 0.65 & & 0.002 & 0.99 & 0.99 \\
\hline
\end{tabular}

$P$-values here refer to the comparison of BCVA and CS at each level of illumination to the preceding one.

vs $1.61 ; P=<0.001,<0.001$ and 0.002$)$. No significant difference was found as the illumination was increased from 200 to 400 lux nor as it was increased from 400 to 1600 lux in either of the two groups (Table 2).

Comparing the multifocal to the monofocal group at the four levels of illumination did not reveal any significant difference in BCVA or CS (Figure 1).

In patients who completed the questionnaire, Table 3 and Figures 2 and 3 showed that all those in the monofocal group, while $81.3 \%$ of those in the multifocal group wore glasses. Also, although there was hardly any difference between the two groups in the lighting preference or the effect of indoor lights on their vision, in the multifocal group $12.5 \%$ experienced blur, $18.8 \%$ experienced glare or halos, and $43.8 \%$ noticed subjective worsening of their vision in bright outdoor lights (sun or headlights).

\section{Discussion}

In this study, we tried to establish if the illumination level would differentially affect the visual acuity and CS in patients with multifocal implants in comparison to those with monofocal implants. Using a range of illumination levels that are commonly used for indoor activities, we found that both visual functions improved with increasing illumination in both groups, which agrees with previous studies on phakic patients, ${ }^{10,12,13}$ and is related to contrast enhancement with increasing illumination.

In both groups, there was no significant improvement in distance or near BCVA or in CS beyond 200 lux, and both groups had comparable visual acuity and CS at the different levels of illumination. These findings are in agreement with many authors, who found no difference between monofocal and multifocal IOLs in distance BCVA. ${ }^{1,3,6,14}$ Also, Schmitz et $a l^{7}$ found no difference in CS between patients with monofocal and those with multifocal IOLs with moderate and strong glare effect. Moreover, Steinert et $a l^{6}$ found no perceived disadvantages attributable to loss of low contrast acuity. In a recent study by Alio et al, although there was a significant difference in CS between groups of patients with monofocal and multifocal IOLs up to 3 months postoperatively, the difference was no longer significant after 6 months and CS improved in both groups. ${ }^{15}$

The questionnaire revealed that the multifocal IOL patients were less likely to wear glasses $(81.3 \%)$ than the monofocal patients $(100 \%)$, which could reflect less dependency of the former group on glasses. It also showed the presence of blur and glare/halo symptoms more commonly in these patients $(12.5 \%$ and $18.8 \%)$ and their absence in the monofocal group, which concur with findings of previous studies. ${ }^{3,4,16}$ In a prospective case series, Dick et al, ${ }^{17}$ found the latter symptoms not to be significantly different in the two groups, and other factors such as the patients' age and corneal surface irregularities were found to have a significant role in causing similar photic phenomena. The subjective appreciation of difficulty with outdoor bright light in the multifocal group further highlights the importance of patient selection. Those who work in bright lighting conditions or frequently drive at night might not be the best candidates for this IOL type.

Although the limited range of illumination used does not represent all the possible illumination levels, we 

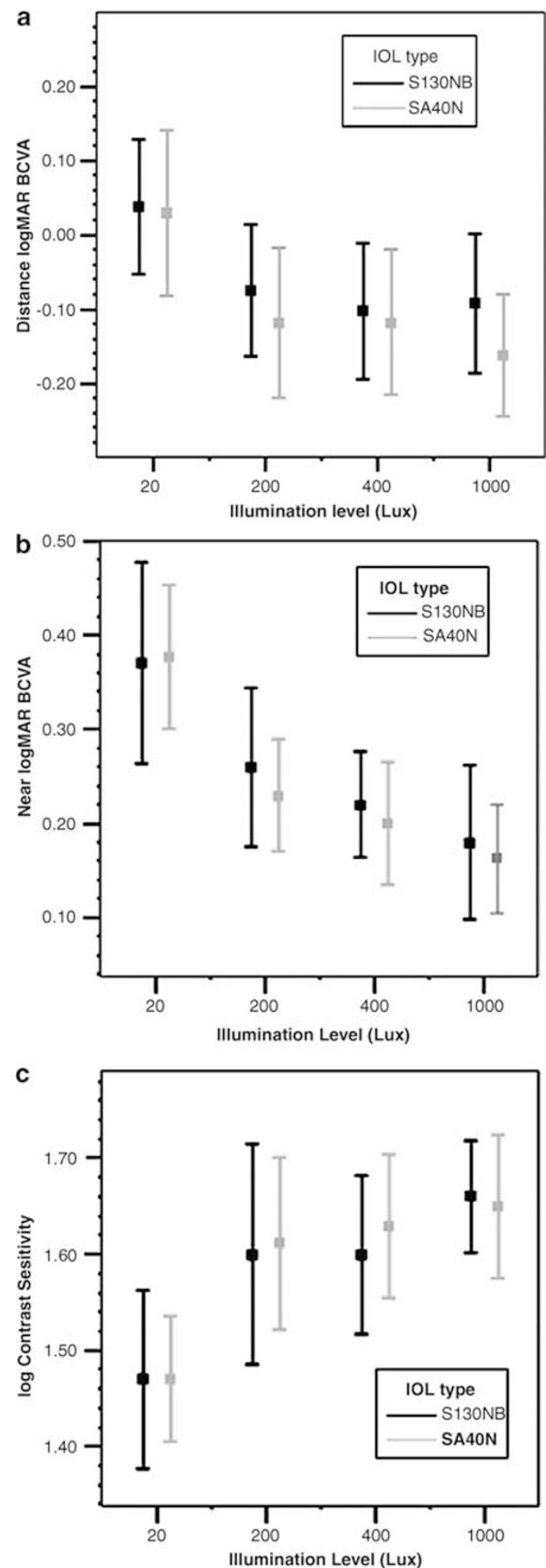

Figure 1 Distance (a) and near (b) BCVA and CS (c) significantly increased with increasing illumination only from 20 to 200 lux in monofocal (SI30NB) and multifocal (SA40N) IOL groups. No significant difference was found between the two groups at the four levels of illumination.
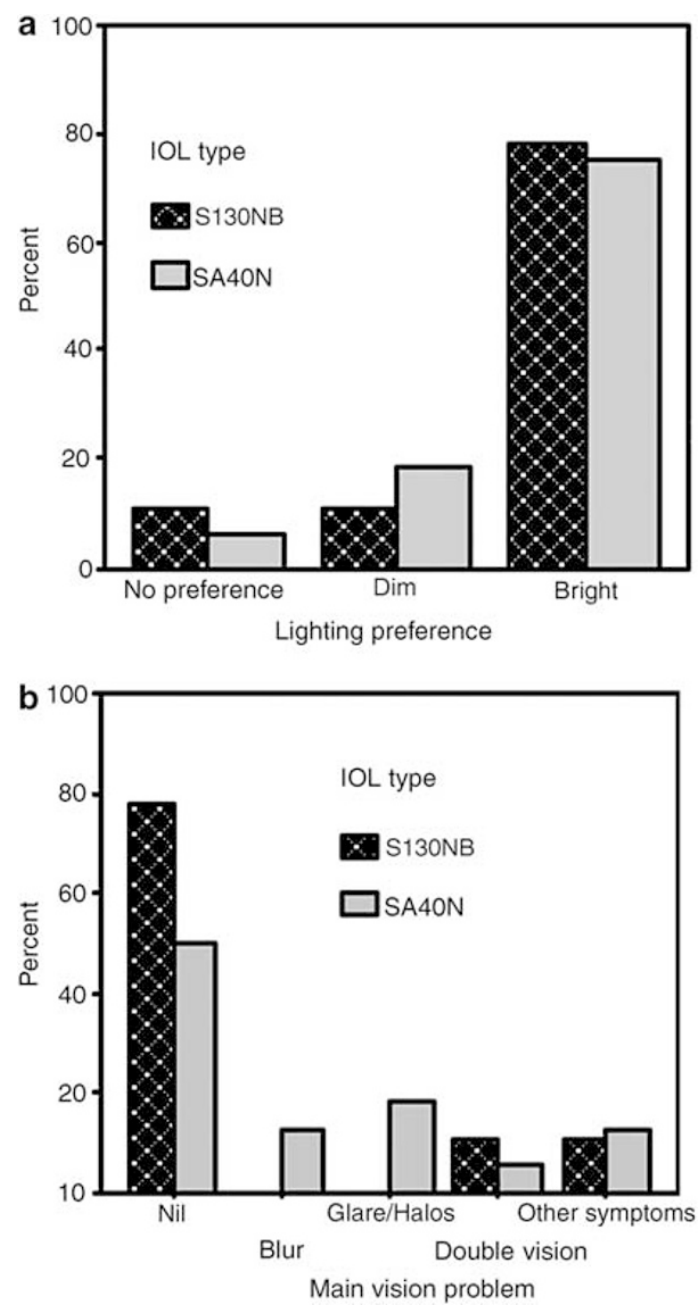

Figure 2 The questionnaire results show that both groups reported similar lighting preferences (a) and that blur and glare/ halo were reported by patients in the multifocal group (b).

chose them to cover the most commonly used indoor levels. We tried to limit the possible IOL selection bias by including consecutive patients and those who had no fundus pathology that can limit the visual function. Future studies should aim to examine a broader spectrum of illumination levels and additional functions such as colour vision, stereopsis and accommodation range.

We conclude that distance and near BCVA and CS improve in patients with monofocal and multifocal IOLs by increasing illumination, but remain comparable in the two groups under common indoor illumination levels. Although glare and halo are more common in patients with multifocal IOLs, they might have only a limited clinical effect on the patients' visual function under the used illumination levels. Outdoor bright illumination seems to negatively affect the visual function in patients with multifocal IOLs. These findings are important for 

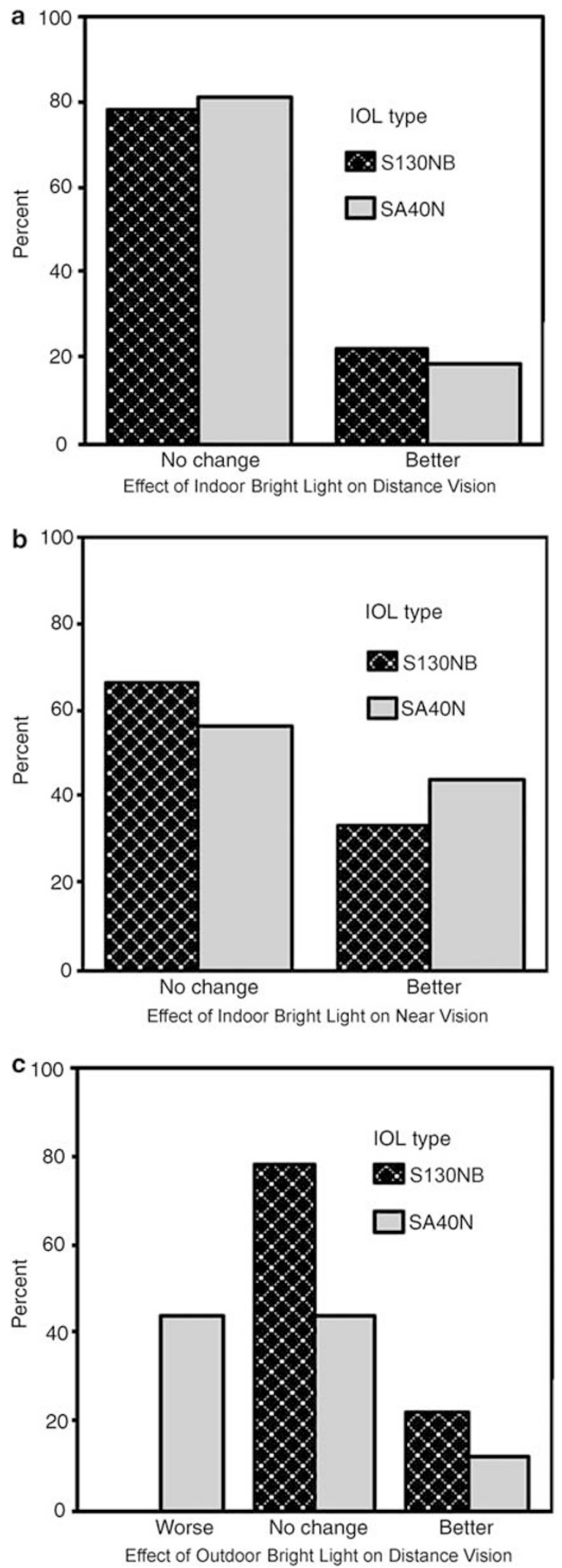

Figure 3 The questionnaire results show that both groups of patients reported that indoor bright light either improved or made no difference to their distance and near vision $(a, b)$ and that more patients in the multifocal group reported that outdoor bright light worsened their vision (c).
Table 3 The questionnaire results

\begin{tabular}{|c|c|c|}
\hline Questions & $\begin{array}{c}\text { Monofocal } \\
(\mathrm{N}=9) \\
\text { No }(\%)\end{array}$ & $\begin{array}{r}\text { Multifoca } \\
(\mathrm{N}=16) \\
\text { No }(\%)\end{array}$ \\
\hline \multicolumn{3}{|l|}{ Do you wear glasses? } \\
\hline Yes & $9(100)$ & 13(81.3) \\
\hline No & 0 & $3(18.8)$ \\
\hline \multicolumn{3}{|l|}{ Are your glasses: } \\
\hline Separate pairs (distance \& or near) & $6(66.7)$ & $5(31.3)$ \\
\hline Bifocals & $2(22.2)$ & $8(50)$ \\
\hline Varifocals & $1(11.1)$ & 0 \\
\hline \multicolumn{3}{|c|}{ Do you have any problem with your vision? } \\
\hline No & 7(77.8) & $8(50)$ \\
\hline Blur & 0 & 2(12.5) \\
\hline Glare/halos? & 0 & $3(18.8)$ \\
\hline Double vision & $1(11.1)$ & $1(6.3)$ \\
\hline Other (please specify) & $1(11.1)^{\mathrm{a}}$ & $2(12.5)^{\mathrm{a}}$ \\
\hline \multicolumn{3}{|l|}{ Do you prefer: } \\
\hline Dim light? & $1(11.1)$ & $3(18.8)$ \\
\hline Bright light? & $7(77.8)$ & $12(75)$ \\
\hline No preference & $1(11.1)$ & $1(6.3)$ \\
\hline
\end{tabular}

How does indoor bright light affect your distance vision?

$\begin{array}{lcc}\text { Makes it worse } & 0 & 0 \\ \text { Makes no change } & 7(77.8) & 13(81.3) \\ \text { Makes it better } & 2(22.2) & 3(18.8)\end{array}$

How does indoor bright light affect your near vision?

$\begin{array}{lcc}\text { Makes it worse } & 0 & 0 \\ \text { Makes no change } & 6(66.7) & 9(56.3) \\ \text { Makes it better } & 3(33.3) & 7(43.8)\end{array}$

How does outdoor bright light (e.g. sunlight/headlights) affect your distance vision?

$\begin{array}{lcc}\text { Makes it worse } & 0 & 7(43.8) \\ \text { Makes no change } & 7(77.8) & 7(43.8) \\ \text { Makes it better } & 2(22.2) & 2(12.5)\end{array}$

How do you rate your distance vision overall?

$\begin{array}{lcc}\text { Poor } & 1(11.1) & 0 \\ \text { Good } & 3(33.3) & 12(75) \\ \text { Excellent } & 5(55.6) & 4(25)\end{array}$

How do you rate your near vision overall?

$\begin{array}{lcc}\text { Poor } & 3(33.3) & 1(6.3) \\ \text { Good } & 4(44.4) & 11(68.8) \\ \text { Excellent } & 2(22.2) & 4(25)\end{array}$

${ }^{\text {aFloaters. }}$

peri-operative counselling and highlight the importance of IOL selection to suit the patient's life style.

\section{Acknowledgements}

We thank Miss Michelle Bradley at the Department of Public Health and Primary Care, University of 
Cambridge for the statistical consultation and advice and Miss Kay Bernacki at Essex County Hospital, Colchester, for her help with the patient recruitment and the study administration.

\section{References}

1 Vaquero-Ruano M, Encinas JL, Millan I, Hijos M, Cajigal C. AMO array multifocal versus monofocal intraocular lenses: long-term follow-up. J Cataract Refract Surg 1998; 24: 118-123.

2 Brydon KW, Tokarewicz AC, Nichols BD. AMO array multifocal lens versus monofocal correction in cataract surgery. J Cataract Refract Surg 2000; 26: 96-100.

3 Javitt J, Brauweiler HP, Jacobi KW, Klemen U, Kohnen S, Quentin CD et al. Cataract extraction with multifocal intraocular lens implantation: clinical, functional, and quality-of-life outcomes. Multicenter clinical trial in Germany and Austria. J Cataract Refract Surg 2000; 26 : 1356-1366.

4 Steinert RF. Visual outcomes with multifocal intraocular lenses. Curr Opin Ophthalmol 2000; 11: 12-21.

5 Leyland MD, Langan L, Goolfee F, Lee N, Bloom PA. Prospective randomised double-masked trial of bilateral multifocal, bifocal or monofocal intraocular lenses. Eye 2002; 16: $481-490$.

6 Steinert RF, Aker BL, Trentacost DJ, Smith PJ, Tarantino N. A prospective comparative study of the AMO ARRAY zonal-progressive multifocal silicone intraocular lens and a monofocal intraocular lens. Ophthalmology 1999; 106: 1243-1255.

7 Schmitz S, Dick HB, Krummenauer F, Schwenn O, Krist R. Contrast sensitivity and glare disability by halogen light after monofocal and multifocal lens implantation. $\mathrm{Br} \mathrm{J}$ Ophthalmol 2000; 84: 1109-1112.
8 Williamson W, Poirier L, Coulon P, Verin P. Compared optical performances of multifocal and monofocal intraocular lenses (contrast sensitivity and dynamic visual acuity). Br J Ophthalmol 1994; 78: 249-251.

9 Haring G, Dick HB, Krummenauer F, Weissmantel U, Kroncke W. Subjective photic phenomena with refractive multifocal and monofocal intraocular lenses, results of a multicenter questionnaire. J Cataract Refract Surg 2001; 27: 245-249.

10 Hecht S. Relation between visual acuity and illumination. J Gen Physiol 1928; 255-281.

11 Williams B. Footcandles and lux for architechtural lighting (An introduction to illuminance). I.E.S. Recommendations 1999. www.mts.net/ william5/library/ illum.htm.

12 Lythgoe RJ. The Measurement of Visual Acuity. Medical Research Council. Special Report Series. London: H.M.Stationary, 1932.

13 Sheedy JE, Bailey IL, Raasch TW. Visual acuity and chart luminance. Am J Optom Physiol Opt 1984; 61: 595-600.

14 Arens B, Freudenthaler N, Quentin CD. Binocular function after bilateral implantation of monofocal and refractive multifocal intraocular lenses. J Cataract Refract Surg 1999; 25: 399-404.

15 Montes-Mico R, Alio JL. Distance and near contrast sensitivity function after multifocal intraocular lens implantation. J Cataract Refract Surg 2003; 29 703-711.

16 Leyland M, Zinicola E. Multifocal versus monofocal intraocular lenses in cataract surgery: a systematic review. Ophthalmology 2003; 110: 1789-1798.

17 Dick HB, Krummenauer F, Schwenn O, Krist R, Pfeiffer N. Objective and subjective evaluation of photic phenomena after monofocal and multifocal intraocular lens implantation. Ophthalmology 1999; 106: 1878-1886. 